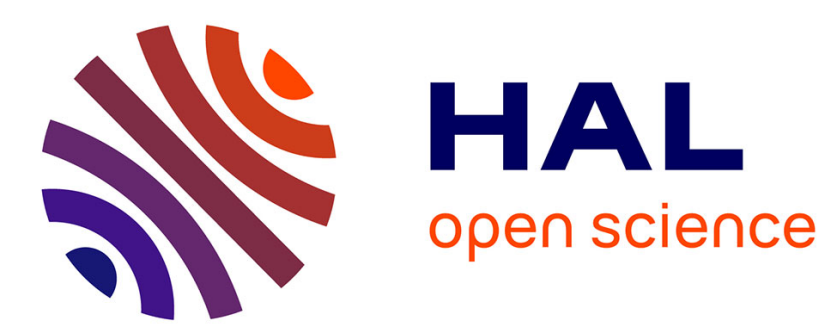

\title{
Elliptic equations with diffusion parameterized by the range of nonlocal interactions
}

\author{
Armel Andami Ovono, Arnaud Rougirel
}

\section{To cite this version:}

Armel Andami Ovono, Arnaud Rougirel. Elliptic equations with diffusion parameterized by the range of nonlocal interactions. 2008. hal-00279310v2

\section{HAL Id: hal-00279310 \\ https://hal.science/hal-00279310v2}

Preprint submitted on 24 Jun 2008

HAL is a multi-disciplinary open access archive for the deposit and dissemination of scientific research documents, whether they are published or not. The documents may come from teaching and research institutions in France or abroad, or from public or private research centers.
L'archive ouverte pluridisciplinaire HAL, est destinée au dépôt et à la diffusion de documents scientifiques de niveau recherche, publiés ou non, émanant des établissements d'enseignement et de recherche français ou étrangers, des laboratoires publics ou privés. 


\title{
ELLIPTIC EQUATIONS WITH DIFFUSION PARAMETERIZED BY THE RANGE OF NONLOCAL INTERACTIONS
}

\author{
ARMEL ANDAMI OVONO \& ARNAUD ROUGIREL
}

\begin{abstract}
We consider quasilinear elliptic equations where the diffusion at each point depends on all the values of the solution in a neighborhood of this point. The size of this neighborhood is parameterized by some non negative number which represents the range of nonlocal interactions. For fixed values of the parameter, the issue of the existence and local uniqueness of the solution is addressed. In a radial symmetric setting, we give pointwise estimates of the solutions and prove the existence of multiple solutions. Regarding bifurcation theory, we show that many local branches of solutions may exist while, among them, only one is global and has no bifurcation point.
\end{abstract}

\section{INTRODUCTION}

Let $\Omega$ be a non empty open bounded subset of $\mathbb{R}^{n}$ whose diameter is denoted by $L$. Let also $g: \Omega \times \Omega \rightarrow \mathbb{R}$ be a measurable function such that

$$
\underset{x \in \Omega}{\operatorname{ess} \sup } \int_{\Omega} g^{2}(x, y) \mathrm{d} y<\infty
$$

and

$$
f \in H^{-1}(\Omega) \text {. }
$$

For any $x$ in $\Omega$ and $r$ in $[0, \infty)$, we define the nonlocal functional

$$
\ell_{r}(\cdot)(x): L^{2}(\Omega) \rightarrow \mathbb{R}, \quad u \mapsto \ell_{r}(u)(x)=\int_{\Omega \cap B(x, r)} g(x, y) u(y) \mathrm{d} y,
$$

where $B(x, r)$ is the closed ball of $\mathbb{R}^{n}$ with radius $r$ and centered at $x$. We notice that $\ell_{0}=0$ and $\ell_{r}=\ell_{L}$ for all $r \geq L$, hence we will often restrict our attention to the case where $r$ belongs to $[0, L]$. In this paper we will investigate the properties of the solutions to the nonlocal problem

$$
(\mathrm{P}-r)\left\{\begin{array}{l}
-\operatorname{div}\left(a\left(\ell_{r}(u)\right) \nabla u\right)=f \quad \text { in } H^{-1}(\Omega) \\
u \in H_{0}^{1}(\Omega)
\end{array}\right.
$$

where the diffusion coefficient $a$ satisfies

$$
a \in C(\mathbb{R}, \mathbb{R}), \quad \inf _{\mathbb{R}} a>0, \quad \sup _{\mathbb{R}} a<\infty .
$$

Date: June 24, 2008.

2000 Mathematics Subject Classification. 35J60, 35B30.

Key words and phrases. Nonlocal elliptic equations, branches of solutions, parameter, nonlocal diffusion. 
If Lipschitz regularity is needed, we will assume instead

$$
a \in W^{1, \infty}(\mathbb{R}), \quad \inf _{\mathbb{R}} a>0 .
$$

If $g \equiv 1$ then $\ell_{r}(u)(x)=\int_{\Omega \cap B(x, r)} u(y) \mathrm{d} y$. In case where $(\mathrm{P}-r)$ modelizes migration of population, it means that the diffusion at $x$ depends on the population surrounding $x$. In the general case, the kernel $g$ accounts for the fact that the contribution to the diffusion process of points in the ball $B(x, r)$ may be different.

The following examples modelize various diffusion process.

(i) $g(x, y)=g(x-y)$ where $g \in L_{l o c}^{2}\left(\mathbb{R}^{n}\right)$. If, for instance $g(x-y)=g(|x-y|)$ is a radial decreasing function then the contribution of a point in $B(x, r)$ will decrease with respect to its distance from $x$. In that case, (1.1) holds since

$$
\int_{\Omega} g^{2}(x-y) \mathrm{d} y=\int_{x-\Omega} g^{2}(y) \mathrm{d} y \leq \int_{B(0,2 R)} g^{2}(y) \mathrm{d} y,
$$

where $R$ is chosen so that $\Omega \subset B(0, R)$.

(ii) $g(x, y)=g(y)$ where $g \in L^{2}(\Omega)$. In particular, being given some subdomain $\Omega^{\prime}$ of $\Omega$, we may choose

$$
g(y)=\mathbb{I}_{\Omega^{\prime}}(y):= \begin{cases}1 & \text { if } y \in \Omega^{\prime} \\ 0 & \text { otherwise }\end{cases}
$$

This model allows to fix which areas of $\Omega$ contribute specifically to the diffusion. If $r=0$ then (P-0) is a linear local elliptic problem. In the case $r=L,(\mathrm{P}-L)$ is a purely nonlocal problem and $\ell_{L}$ is the continuous linear form given by

$$
\ell_{L}(u)=\int_{\Omega} g(y) u(y) \mathrm{d} y \quad \forall u \in L^{2}(\Omega) .
$$

The parameter $r$ measures the range of the nonlocal interactions occurring in the diffusion process. Here, (1.1) is also satisfied.

(iii) Of course, we may combine these two models by choosing

$$
g(x, y)=g_{1}(x-y) g_{2}(y),
$$

where $g_{1} \in L_{l o c}^{2}\left(\mathbb{R}^{n}\right)$ and $g_{2} \in L^{2}(\Omega)$ so that (1.1) holds by the Cauchy-Schwarz inequality.

Our very first aim is to describe the changes that occur in the structure of the set of solutions to $(\mathrm{P}-r)$ as the parameter $r$ varies from 0 to $L$. Indeed, in the case (ii) where the kernel $g$ is independent of $x$, M. Chipot and B. Lovat have shown in [CL99] that (P-L) may have, for instance, three solutions. On the other hand, (1.2), (1.4) implies that (P-0) is uniquely solvable. So natural questions arise from bifurcation theory:

(i) Do there exist branches ${ }^{1}$ of solutions starting from the solutions to (P- $\left.L\right)$ ?

(ii) Do there exist bifurcation point for these branches ?

For generic situations (see Corollary 4.2, Theorem 3.2), the answer to (i) is positive; this is a consequence of the implicit function theorem. However, surprisingly,

\footnotetext{
$1_{\text {see Definition } 4.1}$
} 
it does not seem to exist bifurcation point, as suggested by numerical simulations. Let us describe our result related to question (ii) in vague terms (see Theorem 4.1 for a precise statement). If the diffusion coefficient $a$ is flat in some neighborhood of 0 then there is a unique global branch of solutions and it has no bifurcation point. Figure 1 depict a (possible) generic bifurcation diagram.

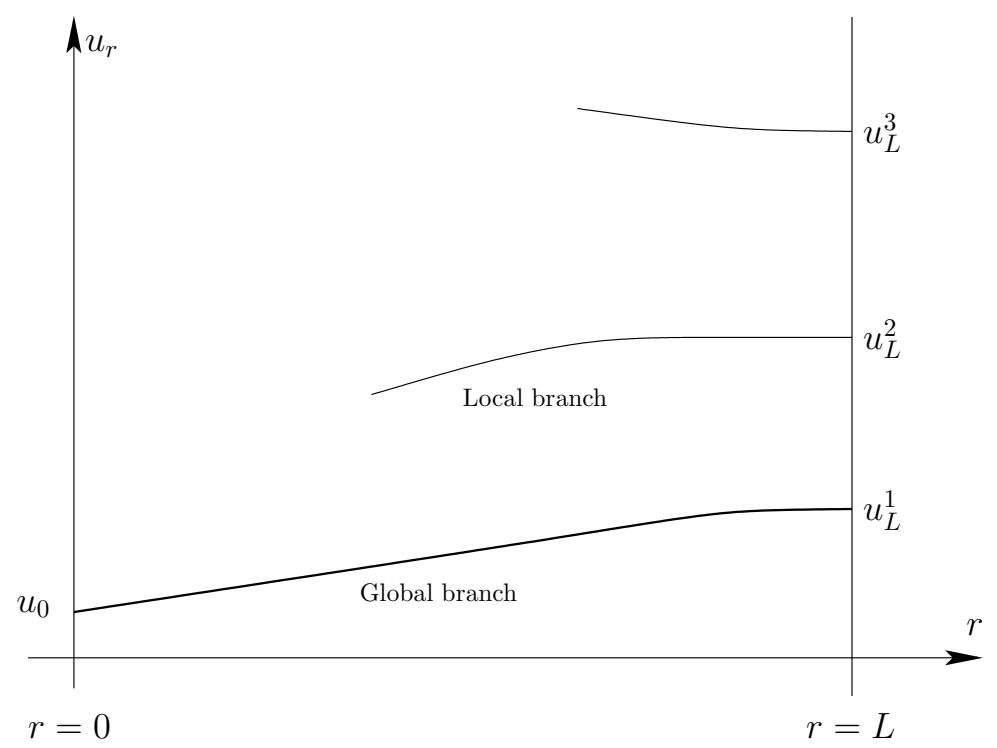

FIGURE 1

It should be emphasize that these results hold for radial symmetric data (see Section 4). Since the structure of nonlocal problems is not so rich than the one of local problems, assumptions have to be strengthened in order to get useful informations about the solutions. This lack of structure may be illustrated as follows. Under assumptions (1.2), (1.4), the local problem

$$
-\operatorname{div}(a(u) \nabla u)=f \quad \text { in } H^{-1}(\Omega), \quad u \in H_{0}^{1}(\Omega),
$$

has a unique solution whereas (P-r) may have infinitely many solutions: see [CM01]. The reader interested with other nonlocal diffusion problems may consult for instance [ZP06] or [CCR06].

This paper is organized as follows. In Section 2, we give some existence and uniqueness results. In particular, Corollary 2.1 states, in implicit function theorem's fashion, the existence of local branches of solutions. Section 3 is devoted to the case where $r=L$ and $g$ is independent of $x$. Preliminary results needed to the study of branches of solutions starting from $r=L$ are given. In particular, Theorem 3.2 characterizes situations where the implicit function theorem may be applied and give, in the case $r=L$, a more powerful result than Corollary 2.1 (which hold in a wider setting). Finally, in section 4, we study radial symmetric solutions. In a first part, some qualitative properties of radial solutions are given. In a second part, we propose some answers to questions (i) and (ii) above. 
If $\mathcal{O}$ is an open part of $\mathbb{R}^{n}$ and $1 \leq p \leq \infty$ then the norm of a function $u$ in $L^{p}(\mathcal{O})$ will be denoted by $\|u\|_{p, \mathcal{O}}$ or simply by $\|u\|_{p}$ if no confusion may occur. The norm in $H^{-1}(\Omega)$ will be denoted by $\|\cdot\|_{*}$.

\section{Existence And Uniqueness RESUlts}

The main result of this section is Corollary 2.1 which is a kind of implicit function Theorem: see Remark 2.1. Theorem 2.1 states that $(\mathrm{P}-r)$ has at least a solution for all non negative parameter $r$. Theorem 2.2 is a local uniqueness result. These results make use of the following Lemmas. If the reader wish to simplify the analysis without losing the essential ideas, he/her may choose $g \equiv 1$.

Lemma 2.1. Under assumption (1.1), for all $u, v$ in $H_{0}^{1}(\Omega)$ and $r, s$ in $[0, L]$, there holds for a.e. $x \in \Omega$,

$$
\left|\ell_{r}(u)(x)-\ell_{s}(v)(x)\right| \leq C_{1} \sup _{\Omega}\|g(x, \cdot)\|_{2}\left(\|\nabla(u-v)\|_{2}+|r-s|^{\frac{1}{n \vee 3}}\|\nabla v\|_{2}\right),
$$

where $C_{1}$ is a constant depending only on $\Omega, n$ and $L ; \sup _{\Omega}\|g(x, \cdot)\|_{2}$ stands for the square root of left-hand side of (1.1) and $n \vee 3$ is the maximum between the dimension $n$ of $\Omega$ and 3 .

Proof. We have

$$
\begin{aligned}
& \left|\ell_{r}(u)(x)-\ell_{s}(v)(x)\right| \leq \\
& \quad \int_{\Omega \cap B(x, r)}|g(x, y)(u-v)(y)| \mathrm{d} y+\int_{\Omega \cap(B(x, s) \backslash B(x, r))}|g(x, y) v(y)| \mathrm{d} y .
\end{aligned}
$$

Let us estimate the latter integral. If $n=1, H_{0}^{1}(\Omega)$ is continuously embedded in $C(\bar{\Omega})$. Then denoting by $C$ the norm of this embedding, it is smaller than

$$
C\|v\|_{\infty} \int_{\Omega}|g(x, y)| \mathbb{1}_{B(x, s) \backslash B(x, r)}(y) \mathrm{d} y \leq C\|v\|_{\infty} \sup _{\Omega}\|g(x, \cdot)\|_{2}|2 s-2 r|^{1 / 2} .
$$

If $n=2$ then we choose $p=6$. If $n \geq 3$, let $p=2 n /(n-2)$. Thus for any $n \geq 2$, by the Hölder inequality and Sobolev's embedding, the latter integral in (2.1) can be estimated by

$$
\begin{aligned}
&\left(\int_{\Omega \cap(B(x, s) \backslash B(x, r))}|g(x, y)|^{p^{\prime}} \mathrm{d} y\right)^{1 / p^{\prime}}\|v\|_{p, \Omega} \leq \\
& C\left(\int_{\Omega}|g(x, y)|^{p^{\prime}} \amalg_{B(x, s) \backslash B(x, r)}(y) \mathrm{d} y\right)^{1 / p^{\prime}}\|\nabla v\|_{2},
\end{aligned}
$$

where $p^{\prime}$ is the conjugate exponent of $p$. Moreover, using again Hölder's inequality with $q:=2 / p^{\prime}$, there holds

$$
\int_{\Omega}|g(x, y)|^{p^{\prime}} \mathbb{I}_{B(x, s) \backslash B(x, r)}(y) \mathrm{d} y \leq\left(\int_{\Omega}|g(x, y)|^{2} \mathrm{~d} y\right)^{1 / q}|B(x, s) \backslash B(x, r)|^{1 / q^{\prime}} .
$$


Since $|B(x, r)|$, the measure of $B(x, r)$ is equal to $c_{n} r^{n}$, we get

$$
\int_{\Omega \cap(B(x, s) \backslash B(x, r))}|g(x, y) v(y)| \mathrm{d} y \leq C_{1}\left(\int_{\Omega}|g(x, y)|^{2} \mathrm{~d} y\right)^{1 / 2}|s-r|^{\frac{1}{p^{\prime} q^{\prime}}}\|\nabla v\|_{2} .
$$

If $n=2, p^{\prime} q^{\prime}=3$ whereas $p^{\prime} q^{\prime}=n$ for $n \geq 3$. Thus for all $n \geq 1$,

$$
\int_{\Omega \cap(B(x, s) \backslash B(x, r))}|g(x, y) v(y)| \mathrm{d} y \leq C_{1} \sup _{\Omega}\|g(x, \cdot)\|_{2}|r-s|^{\frac{1}{n \vee 3}}\|\nabla v\|_{2} .
$$

In the same way,

$$
\int_{\Omega \cap B(x, r)}|g(x, y)(u-v)(y)| \mathrm{d} y \leq C \sup _{\Omega}\|g(x, \cdot)\|_{2}|B(x, r)|^{\frac{1}{n \vee 3}}\|\nabla(u-v)\|_{2} .
$$

Combining these two estimates, going back to (2.1) and using $|B(x, r)| \leq|B(0, L)|$, we obtain the desired estimate.

Lemma 2.2. If (1.1), (1.2), (1.4) hold then for any $(u, v)$ in $H_{0}^{1}(\Omega) \times L^{2}(\Omega)$ satisfying

$$
-\operatorname{div}\left(a\left(\ell_{r}(v)\right) \nabla u\right)=f \quad \text { in } H^{-1}(\Omega)
$$

one has

$$
\|\nabla u\|_{2} \leq \frac{\|f\|_{*}}{\inf _{x \in \Omega} a\left(\ell_{r}(v)\right)(x)} .
$$

Proof. Test the above equation with $u$ and use the continuity of $f$ on $H_{0}^{1}(\Omega)$.

Theorem 2.1. If (1.1), (1.2), (1.4) hold then for all $r$ in $[0, L]$, the problem $(P-r)$ defined by (1.3), has at least one solution.

Proof. We introduce the functional

$$
\begin{aligned}
T_{r}: L^{2}(\Omega) & \rightarrow L^{2}(\Omega) \\
v & \mapsto u, \text { the solution in } H_{0}^{1}(\Omega) \text { to }-\operatorname{div}\left(a\left(\ell_{r}(v)\right) \nabla u\right)=f .
\end{aligned}
$$

Let $M:=k(\Omega)\|f\|_{*} / \inf _{\mathbb{R}} a$ where $k(\Omega)$ is the Poincaré constant. Then Lemma 2.2 implies that $T_{r}$ maps the closed ball of $L^{2}(\Omega)$ with radius $M$ and centered at the origin into itself. Then we conclude by using the Schauder fix point theorem in a standard way.

Theorem 2.2. Under the assumptions (1.1), (1.2), (1.5), let $r_{0} \in[0, L]$ and $u_{r_{0}}$ be a solution to $\left(P-r_{0}\right)$ satisfying

$$
\frac{C_{1} \sup _{\Omega}\|g(x, \cdot)\|_{2}\|f\|_{*}\left\|a^{\prime}\right\|_{\infty}}{\left\{\inf _{x \in \Omega} a\left(\ell_{r_{0}}\left(u_{r_{0}}\right)\right)(x)\right\}^{2}}<1 .
$$

Then there exist $\varepsilon, \delta>0$ such that for any non negative $r \in\left[r_{0}-\varepsilon, r_{0}+\varepsilon\right],(P-r)$ has an unique solution in $B\left(u_{r_{0}}, \delta, H_{0}^{1}(\Omega)\right)$, the closed ball of $H_{0}^{1}(\Omega)$ with radius $\delta$ and centered at $u_{r_{0}}$.

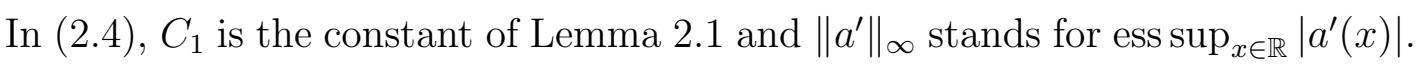


Proof. We apply the Banach fix point theorem in the ball $B\left(u_{r_{0}}, \delta, H_{0}^{1}(\Omega)\right)$ where the value of the radius $\delta$ will be fixed below.

For simplicity, put $K(g):=C_{1} \sup _{\Omega}\|g(x, \cdot)\|_{2}$. Let $\eta_{1}>0$ satisfies the two conditions:

$$
\begin{gathered}
\inf _{\Omega} a\left(\ell_{r_{0}}\left(u_{r_{0}}\right)\right)-\left\|a^{\prime}\right\|_{\infty} \eta_{1}>0 \\
c_{1}:=\frac{K(g)\|f\|_{*}\left\|a^{\prime}\right\|_{\infty}}{\left\{\inf _{\Omega} a\left(\ell_{r_{0}}\left(u_{r_{0}}\right)\right)-\left\|a^{\prime}\right\|_{\infty} \eta_{1}\right\}^{2}}<1 .
\end{gathered}
$$

The existence of such number $\eta_{1}$ is insured by (1.5) and (2.4). Let also $\varepsilon>0$ be such that

$$
K(g) \frac{q(\varepsilon) c_{2}}{1-c_{1}}+q(\varepsilon) K(g)\left\|\nabla u_{r_{0}}\right\|_{2} \leq \eta_{1},
$$

where $q(r):=|r|^{1 / n \vee 3}$ for all $r \in \mathbb{R}$ and

$$
c_{2}:=\frac{K(g)\|f\|_{*}\left\|a^{\prime}\right\|_{\infty}\left\|\nabla u_{r_{0}}\right\|_{2}}{\left\{\inf _{\Omega} a\left(\ell_{r_{0}}\left(u_{r_{0}}\right)\right)-\left\|a^{\prime}\right\|_{\infty} \eta_{1}\right\}^{2}} .
$$

Put $\delta:=q(\varepsilon) c_{2} /\left(1-c_{1}\right)$. Then, for any non negative $r \in\left[r_{0}-\varepsilon, r_{0}+\varepsilon\right]$ and $v$ in $B\left(u_{r_{0}}, \delta, H_{0}^{1}(\Omega)\right)$, the function $u:=T_{r}(v)$ (see (2.3) for the definition of $T_{r}$ ) satisfies

$$
\int_{\Omega} a\left(\ell_{r}(v)\right) \nabla u \nabla \varphi \mathrm{d} x=\int_{\Omega} a\left(\ell_{r_{0}}\left(u_{r_{0}}\right)\right) \nabla u_{r_{0}} \nabla \varphi \mathrm{d} x
$$

for each test function $\varphi$ in $H_{0}^{1}(\Omega)$. The choice $\varphi=u-u_{r_{0}}$ yields

$$
\begin{aligned}
& \inf _{\Omega} a\left(\ell_{r_{0}}\left(u_{r_{0}}\right)\right) \int_{\Omega}\left|\nabla\left(u-u_{r_{0}}\right)\right|^{2} \mathrm{~d} x \leq \\
& \qquad \int_{\Omega}\left|a\left(\ell_{r}(v)\right)-a\left(\ell_{r_{0}}\left(u_{r_{0}}\right)\right)\right||\nabla u|\left|\nabla\left(u-u_{r_{0}}\right)\right| \mathrm{d} x .
\end{aligned}
$$

Let $\eta:=K(g)\left(\delta+q\left(r-r_{0}\right)\left\|\nabla u_{r_{0}}\right\|_{2}\right)$. By Lemma 2.1,

$$
\left|\ell_{r}(v)(x)-\ell_{r_{0}}\left(u_{r_{0}}\right)(x)\right| \leq \eta .
$$

Thus, with (1.5) and the Cauchy-Schwarz inequality

$$
\left\|\nabla\left(u-u_{r_{0}}\right)\right\|_{2} \leq \frac{\left\|a^{\prime}\right\|_{\infty}\|\nabla u\|_{2}}{\inf _{\Omega} a\left(\ell_{r_{0}}\left(u_{r_{0}}\right)\right)} \eta .
$$

Moreover, since $\eta \leq \eta_{1}$ due to the properties of $q$,

$$
\inf _{\Omega} a\left(\ell_{r}(v)\right) \geq \inf _{\Omega} a\left(\ell_{r_{0}}\left(u_{r_{0}}\right)\right)-\left\|a^{\prime}\right\|_{\infty} \eta_{1} .
$$

In view of Lemma 2.2 and (2.9), we have

$$
\begin{aligned}
\left\|\nabla\left(u-u_{r_{0}}\right)\right\|_{2} & \leq \frac{\|f\|_{*}\left\|a^{\prime}\right\|_{\infty}}{\inf _{\Omega} a\left(\ell_{r_{0}}\left(u_{r_{0}}\right)\right)\left\{\inf _{\Omega} a\left(\ell_{r_{0}}\left(u_{r_{0}}\right)\right)-\left\|a^{\prime}\right\|_{\infty} \eta_{1}\right\}} \eta \\
& \leq c_{1} \delta+q(\varepsilon) c_{2} \quad\left(\text { since } q\left(r-r_{0}\right) \leq q(\varepsilon)\right) \\
& =\delta
\end{aligned}
$$


by definition of $\delta$. Hence $T_{r}$ maps $B\left(u_{r_{0}}, \delta, H_{0}^{1}(\Omega)\right)$ into itself. Let us prove that $T_{r}$ is a contraction in this ball. For this consider $v_{1}, v_{2}$ in $B\left(u_{r_{0}}, \delta, H_{0}^{1}(\Omega)\right)$ and put $u_{i}=T_{r}\left(v_{i}\right)$ for $i=1,2$. Arguing as in (2.8), using Lemma 2.1 and recalling that $K(g):=C_{1} \sup _{\Omega}\|g(x, \cdot)\|_{2}$, we get

$$
\begin{aligned}
\inf _{\Omega} a\left(\ell_{r}\left(v_{2}\right)\right) \int_{\Omega} \mid \nabla & \left.\left(u_{1}-u_{2}\right)\right|^{2} \mathrm{~d} x \\
& \leq \int_{\Omega}\left|a\left(\ell_{r}\left(v_{1}\right)\right)-a\left(\ell_{r}\left(v_{2}\right)\right)\right|\left|\nabla u_{1}\right|\left|\nabla\left(u_{1}-u_{2}\right)\right| \mathrm{d} x \\
& \leq K(g)\left\|a^{\prime}\right\|_{\infty}\left\|\nabla\left(v_{1}-v_{2}\right)\right\|_{2}\left\|\nabla u_{1}\right\|_{2}\left\|\nabla\left(u_{1}-u_{2}\right)\right\|_{2} .
\end{aligned}
$$

Thus, thanks to Lemma 2.2,

$$
\left\|\nabla\left(u_{1}-u_{2}\right)\right\|_{2} \leq \frac{K(g)\|f\|_{*}\left\|a^{\prime}\right\|_{\infty}}{\inf _{\Omega} a\left(\ell_{r}\left(v_{1}\right)\right) \inf _{\Omega} a\left(\ell_{r}\left(v_{2}\right)\right)}\left\|\nabla\left(v_{1}-v_{2}\right)\right\|_{2} .
$$

By (2.9), the above coefficient is uniformly bounded by

$$
\frac{K(g)\|f\|_{*}\left\|a^{\prime}\right\|_{\infty}}{\left\{\inf _{\Omega} a\left(\ell_{r_{0}}\left(u_{r_{0}}\right)\right)-\left\|a^{\prime}\right\|_{\infty} \eta_{1}\right\}^{2}}
$$

which is strictly less than 1 by (2.6). Hence $T_{r}$ is a contraction. We conclude by using the Banach fix point theorem.

Let us denote by $F$ the nonlinear operator

$$
\begin{aligned}
F:[0, \infty) \times H_{0}^{1}(\Omega) & \rightarrow H^{-1}(\Omega) \\
(r, u) & \mapsto-\operatorname{div}\left(a\left(\ell_{r}(u)\right) \nabla u\right)-f .
\end{aligned}
$$

Clearly, $F(r, v)=0$ means that $v$ is solution to $(\mathrm{P}-r)$.

Corollary 2.1. Under the assumptions and notation of Theorem 2.2 (in particular $u_{r_{0}}$ denotes a solution to $\left(P-r_{0}\right)$ satisfying $\left.(2.4)\right)$, there exists a Hölder continuous function $u:\left[\left(r_{0}-\varepsilon\right) \vee 0, r_{0}+\varepsilon\right] \rightarrow H_{0}^{1}(\Omega)$ such that

$$
F(r, v)=0 \quad \text { in }\left[\left(r_{0}-\varepsilon\right) \vee 0, r_{0}+\varepsilon\right] \times B\left(u_{r_{0}}, \delta, H_{0}^{1}(\Omega)\right)
$$

if and only if $v=u(r)$.

Remark 2.1. This result is a non smooth version of the implicit function theorem. Indeed, if $a^{\prime}$ is continuous and, for a.e. $x \in \Omega, g(x, \cdot)$ can be extended outside of $\Omega$ by a function lying in $H^{1}\left(\mathbb{R}^{n}\right)$ then $F$ is of class $C^{1}$ and the implicit function theorem may be applied to give a differentiable curve of solutions $r \mapsto u(r)$. In our setting, $F$ is not necessarily differentiable with respect to $u$ (because $a^{\prime} \in L^{\infty}(\mathbb{R})$ ), however we obtain a Hölder continuous branch of solutions through $u_{r_{0}}$ (with Hölder exponent $1 / n \vee 3)$.

Proof of Corollary 2.1. The existence of the function $u$ follows from Theorem 2.2, hence there remains to prove its Hölder regularity. For this, let $\varepsilon, \delta$ be as in the statement of Theorem 2.2 and $r, s$ in $\left[\left(r_{0}-\varepsilon\right) \vee 0, r_{0}+\varepsilon\right]$. Let, as above, $K(g):=C_{1} \sup _{\Omega}\|g(x, \cdot)\|_{2}, q(r)=r^{1 / n \vee 3}$ and $u(r), u(s)$ be the unique solution to 
$(\mathrm{P}-r)$ and $(\mathrm{P}-s)$ in $B\left(u_{r_{0}}, \delta, H_{0}^{1}(\Omega)\right)$. Then arguing as in $(2.10),\|\nabla(u(r)-u(s))\|_{2}$ is estimated by

$$
\frac{K(g)\|f\|_{*}\left\|a^{\prime}\right\|_{\infty}}{\inf _{\Omega} a\left(\ell_{r}\left(u_{r}\right)\right) \inf _{\Omega} a\left(\ell_{s}\left(u_{s}\right)\right)}\left(\|\nabla(u(r)-u(s))\|_{2}+q(r-s)\|\nabla u(s)\|_{2}\right) .
$$

By (2.9), (2.6) and Lemma 2.2,

$$
\left(1-c_{1}\right)\|\nabla(u(r)-u(s))\|_{2} \leq q(r-s) \frac{K(g)\|f\|_{*}^{2}\left\|a^{\prime}\right\|_{\infty}}{\left\{\inf _{\Omega} a\left(\ell_{r_{0}}\left(u_{r_{0}}\right)-\left\|a^{\prime}\right\|_{\infty} \eta_{1}\right\}^{3}\right.},
$$

which shows the Hölder continuity of $u(\cdot)$.

Corollary 2.2. Under the assumptions (1.1), (1.2) and (1.5), the following propositions hold true.

(i) If $r$ is small enough then $(P-r)$ has an unique solution.

(ii) If

$$
\frac{C_{1} \sup _{\Omega}\|g(x, \cdot)\|_{2}\|f\|_{*}\left\|a^{\prime}\right\|_{\infty}}{\inf _{\mathbb{R}} a^{2}}<1
$$

then $(P-r)$ has an unique solution for all $r$ in $[0, L]$.

Proof. The existence follows from Theorem 2.1. The uniqueness is obtained from (2.11) with $u_{i}=v_{i}$. Indeed (2.13) is clearly a sufficient condition. Moreover (see $(2.2)), K(g)$ may be replaced in $(2.11)$ by $C_{1} \sup _{\Omega}\|g(x, \cdot)\|_{2}|B(0, r)|^{1 / n \vee 3}$. Hence, we get the uniqueness for small $r$.

For specific diffusion coefficients $a$, the local uniqueness result of Theorem 2.2 may be applied for $r_{0}:=L$ while multiple solutions to $(\mathrm{P}-L)$ exist: see Remark 3.1. The following theorem gives another local uniqueness result. The main difference with Theorem 2.2 concerns (i) the assumptions on $a^{\prime}$ : it is assumed that we control $a^{\prime}$ only a some neighborhood of 0 (see $(2.15)$ ); (ii) the fact that we obtain a uniqueness result in some tube of $[0, L] \times H_{0}^{1}(\Omega)$ with uniform section (in Theorem 2.1, $\delta$ depends a priori on $r_{0}$ ).

Theorem 2.3. Under the assumptions (1.1), (1.2) and (1.5), assume in addition that

(i) there exists some positive number $\mu_{1}$ such that $a\left(\mu_{1}\right)=\min _{\left[0, \mu_{1}\right]} a$;

(ii) for all $r$ in $[0, L], u_{r}$ is a solution to $(P-r)$ and

$$
0 \leq \ell_{r}\left(u_{r}\right)(x) \leq \mu_{1} \quad \text { for a.e } x \in \Omega ;
$$

(iii) for some number $\delta \mu>0$, there holds

$$
C_{1} \sup _{\Omega}\|g(x, \cdot)\|_{2}\|f\|_{*}\left\|a^{\prime}\right\|_{\infty,\left[-\delta \mu, \mu_{1}+\delta \mu\right]} \frac{1}{a\left(\mu_{1}\right)^{2}}<1 .
$$

Then there exists $\delta>0$ (independent of $r$ and $\left.u_{r}\right)$ such that for all $r \in[0, L],(P-r)$ has an unique solution in $B\left(u_{r}, \delta, H_{0}^{1}(\Omega)\right)$. 
Proof. It is similar to the contractivity-part of the proof of Theorem 2.2. Let $\eta_{1}>0$ satisfies the two conditions:

$$
\begin{aligned}
& a\left(\mu_{1}\right)-\left\|a^{\prime}\right\|_{\infty, I} \eta_{1}>0 \\
& \frac{K(g)\|f\|_{*}\left\|a^{\prime}\right\|_{\infty, I}}{\left\{a\left(\mu_{1}\right)-\left\|a^{\prime}\right\|_{\infty, I} \eta_{1}\right\}^{2}}<1,
\end{aligned}
$$

where $I$ stands for $\left[-\delta \mu, \mu_{1}+\delta \mu\right]$. Let $\delta>0$ satisfies

$$
K(g) \delta \leq \min \left(\delta \mu, \eta_{1}\right) .
$$

Then for any solution $u$ to $(\mathrm{P}-r)$ belonging to $B\left(u_{r}, \delta, H_{0}^{1}(\Omega)\right)$, one has due to Lemma 2.1 and (2.14),

$$
-K(g) \delta \leq \ell_{r}(u) \leq \ell_{r}\left(u_{r}\right)+K(g) \delta .
$$

Thus for a.e. $x \in \Omega, \ell_{r}(u)(x)$ belongs to I owing to (2.18) and (2.14). Hence, (see (2.11)),

$$
\left\|\nabla\left(u-u_{r}\right)\right\|_{2} \leq \frac{K(g)\|f\|_{*}\left\|a^{\prime}\right\|_{\infty, I}}{\inf _{\Omega} a\left(\ell_{r}\left(u_{r}\right)\right) \inf _{\Omega} a\left(\ell_{r}(u)\right)}\left\|\nabla\left(u-u_{r}\right)\right\|_{2} .
$$

Now $\inf _{\Omega} a\left(\ell_{r}\left(u_{r}\right)\right) \geq a\left(\mu_{1}\right)$ by assumptions (i), (ii). Moreover

$$
\begin{aligned}
a\left(\ell_{r}(u)\right) & \geq a\left(\ell_{r}\left(u_{r}\right)\right)-K(g)\left\|a^{\prime}\right\|_{\infty, I} \delta \\
& \geq a\left(\mu_{1}\right)-\left\|a^{\prime}\right\|_{\infty, I} \eta_{1},
\end{aligned}
$$

by (2.18). Hence

$$
\left\|\nabla\left(u-u_{r}\right)\right\|_{2} \leq \frac{K(g)\|f\|_{*}\left\|a^{\prime}\right\|_{\infty, I}}{\left\{a\left(\mu_{1}\right)-\left\|a^{\prime}\right\|_{\infty, I} \eta_{1}\right\}^{2}}\left\|\nabla\left(u-u_{r}\right)\right\|_{2} .
$$

Thus uniqueness follows from (2.17).

3. THE CASE WHERE $r=L$ AND $g$ IS INDEPENDENT OF $x$

In this section, we will assume that $r=L$ and $g$ is independent of $x$, namely

$$
g(x, y)=g(y), \quad g \in L^{2}(\Omega) .
$$

In this case, $\ell_{L}$ becomes a purely nonlocal functional in the sense that it is independent of the spacial variable. We have

$$
\ell_{L}(u)=\int_{\Omega} g(y) u(y) \mathrm{d} y .
$$

Therefore (1.3) reads here

$$
(\mathrm{P}-L)\left\{\begin{array}{l}
-a\left(\ell_{L}(u)\right) \Delta u=f \quad \text { in } H^{-1}(\Omega) \\
u \in H_{0}^{1}(\Omega) .
\end{array}\right.
$$

The solutions to $(\mathrm{P}-L)$ are proportional to the solution $\varphi$ of

$$
-\Delta \varphi=f \quad \text { in } H^{-1}(\Omega), \quad \varphi \in H_{0}^{1}(\Omega) .
$$

More precisely, we may state the following result. 
Theorem 3.1. [CL99] Suppose that (1.2), (1.4) and (3.1) hold. If $u_{L}$ is a solution to $(P-L)$ then

$$
u=\frac{\varphi}{a\left(\ell_{L}\left(u_{L}\right)\right)}
$$

and $\ell_{L}\left(u_{L}\right)$ is solution to

$$
\mu a(\mu)=\ell_{L}(\varphi), \quad \mu \in \mathbb{R} .
$$

Conversely, if $\mu$ is solution to (3.5) then $u=\varphi / a(\mu)$ is solution to $(P-L)$ and $\ell_{L}(u)=\mu$. As a consequence, $(P-L)$ has as much solutions as Equation (3.5).

Remark 3.1. If $u_{L}$ is a solution to (P- $L$ ) then contrary to (2.13), the condition (2.4) which reads here

$$
\frac{C_{1}\|g\|_{2}\|f\|_{*}\left\|a^{\prime}\right\|_{\infty}}{a\left(\ell_{L}\left(u_{L}\right)\right)^{2}}<1
$$

does not imply the uniqueness for $(\mathrm{P}-L)$. Indeed, let $\mu_{1}<\mu_{2}$ be positive numbers. Assuming $\ell_{L}(\varphi)>0$, we consider the Lipschitz continuous function $a$ defined for all $\mu \in \mathbb{R}$ by (see Figure 2)

$$
a(\mu)= \begin{cases}\frac{\ell_{L}(\phi)}{\mu_{1}} & \text { if } \mu \leq \mu_{1} \\ \frac{\ell_{L}(\phi)}{\mu_{2}} & \text { if } \mu \geq \mu_{2} \\ \text { affine } & \text { if } \mu_{1} \leq \mu \leq \mu_{2} .\end{cases}
$$

Then, according to Theorem 3.1, (P-L) possesses two solutions $u_{L}^{1}, u_{L}^{2}$ such that $\ell\left(u_{L}^{1}\right)=\mu_{1}$ and $\ell\left(u_{L}^{2}\right)=\mu_{2}$. Moreover (3.6) reads for $u_{L}=u_{L}^{1}$,

$$
\frac{C_{1}\|g\|_{2}\|f\|_{*} \ell(\varphi)}{\mu_{1} \mu_{2} a\left(\mu_{1}\right)^{2}}<1
$$

and holds true if $\mu_{2}$ is sufficiently large. In what case, Theorem 2.2 yields that $(\mathrm{P}-r)$ has a unique solution in a neighborhood of $u_{L}^{1}$ if $r$ is close enough to $L$.

In order to proceed further, we need to assume that

$$
a \in C^{1}(\mathbb{R}, \mathbb{R}), \quad \inf _{\mathbb{R}} a>0, \quad \sup _{\mathbb{R}} a<\infty .
$$

Let

$$
F: \mathbb{R} \times H_{0}^{1}(\Omega) \rightarrow H^{-1}(\Omega), \quad(r, u) \mapsto-\operatorname{div}\left(a\left(\ell_{r}(u)\right) \nabla u\right)-f,
$$

where $r \mapsto \ell_{r}$ is extended on $(-\infty, 0)$ by setting $\ell_{r}=\ell_{-r}$ for $r<0$. From (3.7), $F$ is continuously differentiable with respect to $u$ and its partial derivative with respect to $u$ is the bounded linear operator defined, for all $v \in H_{0}^{1}(\Omega)$, by

$$
D_{u} F(r, u) v=-\operatorname{div}\left(a\left(\ell_{r}(u)\right) \nabla v\right)-\operatorname{div}\left(a^{\prime}\left(\ell_{r}(u)\right) \ell_{r}(v) \nabla u\right) .
$$

Theorem 3.2. Under assumptions (1.2), (3.1) and (3.7), let $u$ be a solution to $(P-L)$. Then $D_{u} F(r, u)$ is an isomorphism between $H_{0}^{1}(\Omega)$ and $H^{-1}(\Omega)$ if and only if

$$
\ell_{L}(u) a^{\prime}\left(\ell_{L}(u)\right) \neq-a\left(\ell_{L}(u)\right)
$$

Moreover, if (3.9) does not hold then the kernel of $D_{u} F(r, u)$ is the one-dimensional space spanned by $u$. 


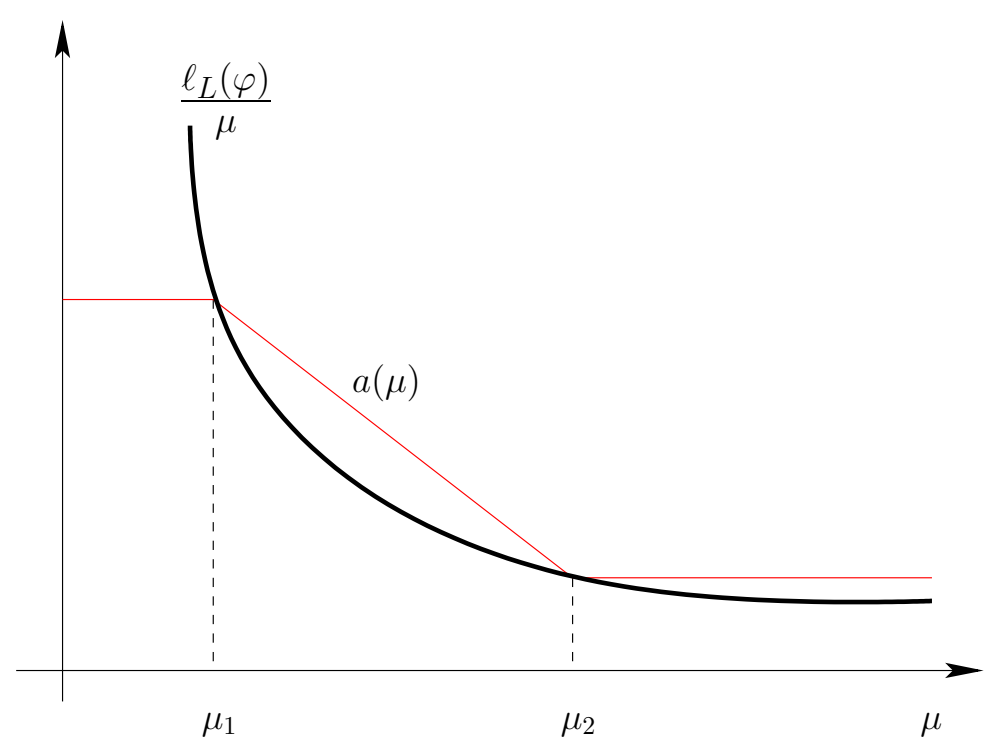

FiguRE 2

Proof. By the Fredholm alternative and the open mapping theorem, $D_{u} F(r, u)$ is an isomorphism if and only if its kernel is trivial. A function $v$ in $H_{0}^{1}(\Omega)$ lies in this kernel if and only if

$$
-\Delta\left(a\left(\ell_{L}(u)\right) v+a^{\prime}\left(\ell_{L}(u)\right) \ell_{L}(v) u\right)=0 \quad \text { in } H^{-1}(\Omega) .
$$

That is to say

$$
v=-\ell_{L}(v) \frac{a^{\prime}\left(\ell_{L}(u)\right)}{a\left(\ell_{L}(u)\right)} u .
$$

Applying $\ell_{L}$ on both side, we obtain

$$
\ell_{L}(v)\left(1+\frac{a^{\prime}\left(\ell_{L}(u)\right)}{a\left(\ell_{L}(u)\right)} \ell_{L}(u)\right)=0 .
$$

Thus (3.9) implies $\ell_{L}(v)=0$. Hence $v=0$ by (3.10) and $D_{u} F(r, u)$ is an isomorphism. If (3.9) is not true that is to say if $\ell_{L}(u) a^{\prime}\left(\ell_{L}(u)\right)=-a\left(\ell_{L}(u)\right)$ then $u \neq 0$ according to (1.4) and the kernel of $D_{u} F(r, u)$ is contained in $\mathbb{R}$.u by (3.10). Since we prove easily that $D_{u} F(r, u) u=0$, these two sets coincide which finish the proof of the theorem.

Corollary 3.1. Under the assumptions (1.2), (3.1) and (3.7), suppose in addition that $g$ can be extended outside of $\Omega$ by a function $\bar{g}$ belonging to $H^{1}\left(\mathbb{R}^{n}\right)$. If each solution to $(P-L)$ satisfies (3.9) then there exists $\varepsilon>0$ with the following property. For all $r$ in $[L-\varepsilon, L],(P-r)$ and $(P-L)$ have the same (finite) number of solutions.

Proof. According to (3.7), the solutions to (3.5) remain in a compact subset of $\mathbb{R}$. Thus if the number of solutions to (3.5) is infinite, there exist $\left(\mu_{n}\right)_{n \geq 0}$ and $\mu$ in $\mathbb{R}$ 
such that $\mu_{n} \rightarrow \mu, \mu_{n} \neq \mu$ and $\mu_{n}, \mu$ solve (3.5) for all $n \geq 0$. As a consequence

$$
\frac{a\left(\mu_{n}\right)-a(\mu)}{\mu_{n}-\mu} \rightarrow a^{\prime}(\mu)
$$

and, using (3.5),

$$
\frac{a\left(\mu_{n}\right)-a(\mu)}{\mu_{n}-\mu}=\frac{\ell_{L}(\varphi) / \mu_{n}-\ell_{L}(\varphi) / \mu}{\mu_{n}-\mu} \rightarrow-\frac{\ell_{L}(\varphi)}{\mu^{2}}=-\frac{a(\mu)}{\mu} .
$$

Thus $a^{\prime}(\mu)=-\frac{a(\mu)}{\mu}$, so $u:=\varphi / a(\mu)$ is, by Theorem 3.1, a solution to (P- $\left.L\right)$ which does not satisfies (3.9) since $\ell_{L}(u)=\mu$. We conclude that under the assumptions of the corollary, $(\mathrm{P}-L)$ has a finite number of solutions.

Let $r>0$ and $u \in H_{0}^{1}(\Omega) ; u$ is extended by zero outside of $\Omega$. Then, for each $x \in \Omega$,

$$
\frac{\partial}{\partial r} \ell_{r}(u)(x)=\int_{\Omega \cap \partial B(x, r)} g \cdot u(\sigma) \mathrm{d} \sigma .
$$

Indeed, if $\bar{g} \cdot u$ is continuous on $\mathbb{R}^{n}$, we have

$$
\begin{aligned}
\frac{\partial}{\partial r} \ell_{r}(u)(x) & =\frac{\partial}{\partial r} \int_{B(x, r)} \bar{g} \cdot u(y) \mathrm{d} y \\
& =\frac{\partial}{\partial r} \int_{\|\sigma\|=1} \int_{0}^{r} \bar{g} \cdot u(x+s \sigma) s^{n-1} \mathrm{~d} s \mathrm{~d} \sigma \\
& =\int_{\|\sigma\|=1} \bar{g} \cdot u(x+r \sigma) r^{n-1} \mathrm{~d} \sigma \\
& =\int_{\Omega \cap \partial B(x, r)} g \cdot u(\sigma) \mathrm{d} \sigma .
\end{aligned}
$$

The general case follows by a density argument.

In particular,

$$
\frac{\partial}{\partial r} \ell_{r}(u)(x)_{r=L}=0
$$

Hence using also (3.7), we may prove that $F$ is $C^{1}$ on $(0, \infty) \times H_{0}^{1}(\Omega)$ and

$$
D_{r} F(r, u)=-\operatorname{div}\left(a^{\prime}\left(\ell_{r}(u)\right) \frac{\partial}{\partial r} \ell_{r}(u) \nabla u\right) .
$$

By Theorem 3.2 and the implicit function theorem, we deduce that for each solution $u_{L}$ to $(\mathrm{P}-L)$, there exists $\delta>0$ such that $(\mathrm{P}-r)$ possesses a unique solution in $B\left(u_{L}, \delta, H_{0}^{1}(\Omega)\right)$. Since $(\mathrm{P}-L)$ has a finite number of solutions, we may conclude with a standard compactness argument that $(\mathrm{P}-r)$ and $(\mathrm{P}-L)$ have the same number of solutions if $r$ is sufficiently close to $L$.

Remark 3.2. Let $u_{L}$ be a solution to (P-L) satisfying (3.9) and $r \mapsto u(r)$ be a branch of solution to $(\mathrm{P}-r)$ starting from $u_{L}$. By the implicit function theorem,

$$
u^{\prime}(L)=-D_{u} F\left(L, u_{L}\right)^{-1} D_{r} F\left(L, u_{L}\right) .
$$


From (3.11), there holds $u^{\prime}(L)=0$ thus Taylor's expansion yields

$$
u(r)=u_{L}+o(r-L) \quad \text { in } H_{0}^{1}(\Omega)
$$

where $o(\varepsilon) / \varepsilon \rightarrow 0$ in $H_{0}^{1}(\Omega)$ as $\varepsilon \rightarrow 0$. In the same way, for $r$ close to zero, assuming $g \equiv 1$ and denoting by $u_{0}=\varphi / a(0)$ the solution to (P-0), we have

$$
u(r)=u_{0}-\delta_{1}(n) \frac{a^{\prime}(0)}{a(0)} r u_{0}^{2}+o(r) \quad \text { in } H_{0}^{1}(\Omega),
$$

where $\delta_{1}(1)=1$ and $\delta_{1}(n)=0$ if $n \geq 1$. Indeed, by the implicit function theorem, $u^{\prime}(0)=-D_{u} F\left(0, u_{0}\right)^{-1} D_{r} F\left(0, u_{0}\right)$ that is to say, using (3.8),

$$
\left.\frac{\partial}{\partial r} \ell_{r}(u)(x)\right|_{r=0}=2 \delta_{1}(n) u(x) \quad \text { a.e. } x \in \Omega
$$

and (3.12), we deduce that $w:=u^{\prime}(0)$ is solution to

$$
-a(0) \Delta w=2 \delta_{1}(n) a^{\prime}(0) \operatorname{div}\left(u_{0} \nabla u_{0}\right)=\delta_{1}(n) a^{\prime}(0) \Delta\left(u_{0}^{2}\right) \quad \text { in } H^{-1}(\Omega) .
$$

Hence $w=-\delta_{1}(n) \frac{a^{\prime}(0)}{a(0)} u_{0}^{2}$ and (3.13) follows.

Remark 3.3. Under the assumptions of Theorem 3.2, $D_{u} F\left(L, u_{L}\right)$ is invertible if and only if the tangents to the graph of $a(\cdot)$ and $\mu \mapsto \ell_{L}(\varphi) / \mu$ are distinct at the point $\mu_{L}=\ell_{L}\left(u_{L}\right)$. Indeed, by Theorem 3.2, $D_{u} F\left(L, u_{L}\right)$ is non invertible if and only if $\mu_{L}$ satisfies

$$
a^{\prime}\left(\mu_{L}\right)=-\frac{a\left(\mu_{L}\right)}{\mu_{L}}
$$

Since $\mu_{L} a\left(\mu_{L}\right)=\ell_{L}(\varphi)$, we deduce that

$$
a^{\prime}\left(\mu_{L}\right)=\frac{\mathrm{d}}{\mathrm{d} \mu}\left(\frac{\ell_{L}(\varphi)}{\mu}\right)_{\mu=\mu_{L}} .
$$

Hence, the graphs of $a(\cdot)$ and $\mu \mapsto \ell_{L}(\varphi) / \mu$ have the same tangent at $\mu_{L}$.

\section{RAdial SOlutions}

This section is devoted to the study of the behaviour of solutions $u_{r}$ to (1.3) as the parameter $r$ ranges from 0 to $L$. More precisely, denoting by $u_{0}, u_{L}$ two solutions of (P-0) and (P-L), we would like to compare $u_{r}$ with $u_{0}$ and $u_{L}$. For instance in the case where $u_{L} \leq u_{0}$, we wish to show that for all $r$ in $[0, L]$,

$$
u_{L} \leq u_{r} \leq u_{0} \text {. }
$$

The main tool for this aim is Proposition 4.2 which state the monotonicity of the solution to elliptic problems with respect to the coefficient of diffusion. This proposition hold in a radial symmetric setting, what is why this section is concerned with radial solutions. A second king of results deals with the existence of local or global branches of solutions and the non existence of bifurcation point: see Theorem 4.1 and Corollary 4.2.

In the sequel, we will assume that $\Omega$ is the open ball of $\mathbb{R}^{n}$ with radius $L / 2$ and centered at zero so that $L$ remains the diameter of $\Omega$. For each function $u$ in $L_{\mathrm{r}}^{2}(\Omega)$, the subspace of $L^{2}(\Omega)$ consisting of radial functions, we will denote by $\tilde{u}$ 
the function of $L^{2}(0, L / 2)$ associated with $u$, namely $\tilde{u}(\|x\|)=u(x)$ for a.e. $x$ in $\Omega$. In this section we will make use of the following assumptions.

$$
\begin{aligned}
& f \in L_{\mathrm{r}}^{2}(\Omega) \\
& g(x, y)=g(y), \quad g \in L_{\mathrm{r}}^{2}(\Omega) \\
& a \in C(\mathbb{R}, \mathbb{R}), \quad \inf _{\mathbb{R}} a>0, \quad \sup _{\mathbb{R}} a<\infty \\
& f \geq 0 \quad \text { a.e. in } \Omega \\
& g \geq 0 \quad \text { a.e. in } \Omega .
\end{aligned}
$$

4.1. A linear problem. For

$$
A \in W^{1, \infty}(\Omega) \text { radial, } \quad \inf _{\Omega} A>0,
$$

consider the linear problem

$$
-\operatorname{div}(A(x) \nabla u)=f \quad \text { in } L^{2}(\Omega), \quad u \in H^{2}(\Omega) \cap H_{0}^{1}(\Omega) .
$$

Proposition 4.1. If (4.1), (4.6) hold then (4.7) has a unique solution u. Moreover it is radial and for a.e. $t$ in $[0, L / 2]$,

$$
\begin{aligned}
-\left(\tilde{A} \tilde{u}^{\prime}\right)^{\prime}-\frac{n-1}{t} \tilde{A} \tilde{u}^{\prime} & =\tilde{f}(t) \\
\tilde{u}^{\prime}(t) & =-\frac{1}{\tilde{A}(t)} \int_{0}^{t}\left(\frac{s}{t}\right)^{n-1} \tilde{f}(s) \mathrm{d} s .
\end{aligned}
$$

Proof. It is well known that (4.7) has a unique radial solution satisfying (4.8). In order to prove (4.9), put $U:=\tilde{A} \tilde{u}^{\prime}$. Then by (4.8),

$$
-U^{\prime}-\frac{n-1}{t} U=\tilde{f}
$$

Hence $\left(U t^{n-1}\right)^{\prime}=-t^{n-1} \tilde{f}$ and (4.9) follows by integration.

Proposition 4.2. Under the assumptions (4.1), (4.4), let $A, B \in C(\bar{\Omega})$ be positive radial functions such that $A \leq B$ in $\bar{\Omega}$. Denote respectively by $u$ and $v$ the solution to

$$
-\operatorname{div}(A(x) \nabla u)=f \quad \text { in } H^{-1}(\Omega), \quad u \in H_{0}^{1}(\Omega)
$$

and

$$
-\operatorname{div}(B(x) \nabla v)=f \quad \text { in } H^{-1}(\Omega), \quad v \in H_{0}^{1}(\Omega) .
$$

Then $u \geq v$ a.e. in $\Omega$.

Proof. First assume that $A$ and $B$ are smooth functions. By (4.9) and $A \leq B$, for each $t$ in $[0, L / 2]$, one has $\tilde{u}^{\prime}(t) \leq \tilde{v}^{\prime}(t)$. Thus, since $\tilde{u}(L / 2)=\tilde{v}(L / 2)=0$,

$$
\tilde{u}(t)=\int_{L / 2}^{t} \tilde{u}^{\prime}(s) \mathrm{d} s \geq \int_{L / 2}^{t} \tilde{v}^{\prime}(s) \mathrm{d} s=\tilde{v}(t) .
$$

Hence $u \geq v$. 
In the general case, let $\varepsilon$ be any positive number such that $\inf _{\Omega} A-\varepsilon>0$. Put $A_{\varepsilon}:=A-\varepsilon$ and denote by $u_{\varepsilon}$ the solution to

$$
-\operatorname{div}\left(A_{\varepsilon}(x) \nabla u_{\varepsilon}\right)=f \quad \text { in } H^{-1}(\Omega), \quad u_{\varepsilon} \in H_{0}^{1}(\Omega) .
$$

Since $A_{\varepsilon}+\varepsilon \leq B$, there exists $A_{n}, B_{n}$ in $C^{1}(\bar{\Omega})$ satisfying

$$
\begin{aligned}
A_{n} \rightarrow A_{\varepsilon}, \quad B_{n} \rightarrow B & & \text { in } C(\bar{\Omega}) \\
0<c \leq A_{n}(x) \leq B_{n}(x) & & \forall x \in \bar{\Omega}, \forall n \geq 0,
\end{aligned}
$$

where $c$ is a constant independent of $n$ and $x$. Let $u_{n}$ and $v_{n}$ be the solutions to the linear problem corresponding to the diffusions $A_{n}$ and $B_{n}$. The first part of this proof yields that $u_{n} \geq v_{n}$. By a compactness argument, we deduce that, up to a subsequence,

$$
u_{n} \underset{n \rightarrow \infty}{\longrightarrow} u_{\varepsilon}, \quad v_{n} \underset{n \rightarrow \infty}{\longrightarrow} v \quad \text { a.e. in } \Omega .
$$

Thus $u_{\varepsilon} \geq v$. Also by the same compactness argument, up to a subsequence, $u_{\varepsilon} \rightarrow u$ as $\varepsilon \rightarrow 0$. Finally, $u \geq v$.

4.2. Back to the nonlocal problem. Let us first investigate the quantitative properties of the set of solutions to $(\mathrm{P}-r)$. For this purpose, wider assumptions on the kernel $g$ may replace (4.2), (4.5), namely, we will assume (1.1) and that $g$ is radial in the following sense: for all rotation $R$ of $\mathbb{R}^{n}$,

$$
g(R x, R y)=g(x, y) \quad \text { for a.e. }(x, y) \in \Omega \times \Omega .
$$

In the sequel, we will apply Proposition 4.2 with $A(x)=a\left(\ell_{r}(u)(x)\right)$. So we need the continuity of $x \mapsto a\left(\ell_{r}(u)(x)\right)$ on $\bar{\Omega}$. This will be insured by the following assumptions on $g$.

$$
\begin{array}{ll}
g(\cdot, y) \in C(\bar{\Omega}) & \text { a.e. } y \in \Omega \\
|g(x, y)| \leq h(y) & \text { a.e. }(x, y) \in \Omega \times \Omega, \quad h \in L^{2}(\Omega) .
\end{array}
$$

In order to control the sign of $u_{r}$, we will assume

$$
g \geq 0 \quad \text { a.e. in } \Omega \times \Omega .
$$

We are now in position to state an existence result for radial solutions.

Proposition 4.3. If (1.1), (4.1), (4.3), (4.10) hold true then (P-r) has at least a radial solution.

Proof. For all $v$ in $L_{\mathrm{r}}^{2}(\Omega), a\left(\ell_{r}(v)\right)$ is radial since it is easily proved that for all rotation $R$ of $\mathbb{R}^{n}, \ell_{r}(v)(R x)=\ell_{r}(v)(x)$ for a.e. $x \in \Omega$. Thus, we can show easily that $T_{r}$ defined by $(2.3)$ maps $L_{\mathrm{r}}^{2}(\Omega)$ into itself. The assertion follows by a fix point argument (see the proof of Theorem 2.1).

We will show in Proposition 4.2 that $(\mathrm{P}-r)$ has multiple solutions for suitable diffusion coefficients. If $\varphi$ is the solution to (3.3) then, for all $r$ in $[0, L]$, we denote by $I_{r}$ the interval

$$
I_{r}:=\left[\inf _{\Omega} \ell_{r}(\varphi), \sup _{\Omega} \ell_{r}(\varphi)\right]
$$


We will make use of the following result which is, in some sense, a generalization of Theorem 3.1 to the case $r \in[0, L]$.

Lemma 4.1. Under the assumptions (4.1), (4.3), (4.4), (4.10)-(4.13), let $r \in[0, L]$ and suppose that the function a has the following properties. There exist $0 \leq m \leq$ $M$ such that

$$
\begin{array}{rlrl}
a(m) & =\max _{[m, M]} a & a(M) & =\min _{[m, M]} a \\
m a(m) & \leq \min I_{r} & \max I_{r} \leq M a(M) .
\end{array}
$$

Then $(P-r)$ admits a radial solution $u$ and

$$
m \leq \ell_{r}(u) \leq M \quad \text { a.e. in } \Omega .
$$

Proof. The set

$$
E:=\left\{u \in L_{\mathrm{r}}^{2}(\Omega): m \leq \ell_{r}(u) \leq M \text { a.e. in } \Omega\right\}
$$

is closed, convex and non empty. Indeed, $\varphi / a(m)$ belongs this set due to (4.14) and (4.15).

By (4.14), for all $v$ in $E, a(m) \geq a\left(\ell_{r}(v)\right) \geq a(M)$. Moreover, $a\left(\ell_{r}(v)\right)$ is radial and belongs to $C(\bar{\Omega})$ due to (4.11), (4.12) and Lebesgue's Theorem. Proposition 4.2 then yields

$$
\frac{\varphi}{a(m)} \leq T_{r}(v) \leq \frac{\varphi}{a(M)}
$$

Since $g$ is non negative,

$$
\frac{\ell_{r}(\varphi)}{a(m)} \leq \ell_{r}\left(T_{r}(v)\right) \leq \frac{\ell_{r}(\varphi)}{a(M)}
$$

Thus $T_{r}(v)$ (defined by (2.3)) belongs to $E$ according to (4.15). We conclude with the Schauder fix point theorem.

Proposition 4.4. Under the assumptions (4.1), (4.3), (4.4), (4.10)-(4.13), let $r \in$ $[0, L]$ and suppose that the function a has the following properties. There exist $0<M_{1}<m_{2}<M_{2}$ such that

$$
\begin{aligned}
& a(0)=\max _{\left[0, M_{1}\right]} a \quad a\left(M_{1}\right)=\min _{\left[0, M_{1}\right]} a \quad \max I_{r} \leq M_{1} a\left(M_{1}\right) \\
& a\left(m_{2}\right)=\max _{\left[m_{2}, M_{2}\right]} a \quad a\left(M_{2}\right)=\min _{\left[m_{2}, M_{2}\right]} a \\
& m_{2} a\left(m_{2}\right) \leq \min I_{r} \quad \max I_{r} \leq M_{2} a\left(M_{2}\right) .
\end{aligned}
$$

Then $(P-r)$ has at least two solutions.

Proof. We apply Lemma 4.1 with $m=0$ and $M=M_{1}$ to get a solution $u$ such that $\ell_{r}(u) \leq M_{1}$ a.e. in $\Omega$. Using again this lemma with $m=m_{2}$ and $M=M_{2}$, we obtain a solution $v$ to $(\mathrm{P}-r)$ with $m_{2} \leq \ell_{r}(v)$. Since $M_{1}<m_{2}, u$ and $v$ are distinct solutions. 
Remark 4.1. There exists functions a satisfying the conditions of Proposition 4.4. Indeed, let $r \in(0, L], a(0)>0$ and $M_{1}:=2 \max I_{r} / a(0)$. We put

$$
a\left(M_{1}\right):=\frac{a(0)}{2}
$$

and suppose that $a$ is decreasing on $\left[0, M_{1}\right]$. Thus (4.16) holds. Besides, suppose that $f, g$ satisfy the assumptions of Lemma 4.1 together with $f \not \equiv 0$ and $g>0$ a.e. in $\bar{\Omega} \times \Omega$. Then $\min I_{r}>0$ by the strong maximum principle. Let $m_{2}>$ $M_{1}$ and define $a\left(m_{2}\right):=\min I_{r} / m_{2}$. Finally, let $M_{2}>m_{2}$ be such that $M_{2}:=$ $2 \max I_{r} / a\left(m_{2}\right)$ and define $a\left(M_{2}\right):=a\left(m_{2}\right) / 2$. We have

$$
m_{2} a\left(m_{2}\right)=\min I_{r} \leq \max I_{r}=M_{2} \frac{a\left(m_{2}\right)}{2}=M_{2} a\left(M_{2}\right) .
$$

We suppose that $a$ is decreasing on $\left[m_{2}, M_{2}\right]$. Hence $m_{2}, M_{2}$ fit also the assumptions of the proposition.

Clearly, this procedure may be repeated to construct diffusion coefficient a leading to the existence of any finite number of solutions for $(\mathrm{P}-r)$.

Let us investigate the qualitative properties of radial solutions.

Proposition 4.5. Under the assumptions (4.1), (4.3), (4.4), (4.10)-(4.13), let $\varphi$ be the solution to (3.3). Then, for any $r$ in $[0, L]$ and any radial solution $u_{r}$ to $(P-r)$, one has

$$
\frac{\varphi}{\sup _{[0, \infty)} a} \leq u_{r} \leq \frac{\varphi}{\inf _{[0, \infty)} a} \quad \text { a.e in } \Omega
$$

Proof. Since $f, g, u_{r}$ are non negative, the following trivial estimates hold

$$
\inf _{[0, \infty)} a \leq a\left(\ell_{r}\left(u_{r}\right)\right) \leq \sup _{[0, \infty)} a .
$$

Proposition 4.2 yields the result.

Until the end of this paper, we will focus on the case where $g$ is independent of $x$. By Theorem 3.1, the solutions to $(\mathrm{P}-0)$ and $(\mathrm{P}-L)$ are ordered. More precisely, $u_{0}=\varphi / a(0)$ is solution to $(\mathrm{P}-0)$ and any solution $u_{L}$ to $(\mathrm{P}-L)$ satisfies $u_{L}=\varphi / a\left(\mu_{L}\right)$ for some solution $\mu_{L}$ to (3.5). Taking into account the monotonicity of $u \mapsto \ell_{r}(u)$, we obtain different type of results according to whether $a(0) \leq a\left(\mu_{L}\right)$ or not. We will first assume that $a(0) \leq a\left(\mu_{L}\right)$ so that $u_{L} \leq u_{0}$.

Corollary 4.1. Under assumptions (4.1)-(4.5), assume in addition that the function a has the following property. There exits a solution $\mu_{L}$ to (3.5) such that $a\left(\mu_{L}\right)=\sup _{[0, \infty)} a$ and $a(0)=\inf _{[0, \infty)} a$. Then $u_{L}=\varphi / a\left(\mu_{L}\right)$ is solution to $(P-L)$ and any radial solution $u_{r}$ to $(P-r)$ satisfies

$$
u_{L} \leq u_{r} \leq u_{0} .
$$

Proof. We have $u_{0}=\varphi / \inf _{[0, \infty)} a$. Moreover, according to Theorem 3.1, $u_{L}$ is solution to $(\mathrm{P}-L)$ thus the assertion follows from Proposition 4.5.

Proposition 4.6. Under the assumptions (4.1)-(4.5), consider any parameter $r$ in $[0, L]$ and assume the following. 
(i) There exits a solution $\mu_{L}$ to (3.5) such that $a\left(\mu_{L}\right)=\max _{\left[0, \mu_{L}\right]}$ a and

$$
\sup _{x \in \Omega} \ell_{r}(\varphi)(x) \leq a(0) \mu_{L}
$$

(ii) $a(0)=\min _{\left[0, \mu_{L}\right]} a$.

Then there exists a solution $u_{r}$ to $(P-r)$ with $u_{L} \leq u_{r} \leq u_{0}$ a.e. in $\Omega\left(u_{L}:=\right.$ $\left.\varphi / a\left(\mu_{L}\right)\right)$.

Proof. Define $\bar{a}: \mathbb{R} \rightarrow \mathbb{R}$ by

$$
\bar{a}(\mu)= \begin{cases}a(\mu) & \text { if } 0 \leq \mu \leq \mu_{L} \\ a(0) & \text { if } \mu \leq 0 \\ a\left(\mu_{L}\right) & \text { if } \mu_{L} \leq \mu\end{cases}
$$

and, in view of Proposition 4.3, consider a solution $\bar{u}_{r}$ of

$$
-\operatorname{div}\left(\bar{a}\left(\ell_{r}(u)\right) \nabla u\right)=f \quad \text { in } L^{2}(\Omega), \quad u \in H^{2}(\Omega) \cap H_{0}^{1}(\Omega) \cap L_{\mathrm{r}}^{2}(\Omega) .
$$

By Corollary 4.1 and the definition of $\bar{a}, u_{L} \leq \bar{u}_{r} \leq u_{0}$. Since $u_{L}, g \geq 0$,

$$
0 \leq \ell_{r}\left(\bar{u}_{r}\right) \leq \ell_{r}\left(u_{0}\right)=\frac{\ell_{r}(\varphi)}{a(0)} \leq \mu_{L}
$$

according to (4.17). Therefore $\bar{a}\left(\ell_{r}\left(\bar{u}_{r}\right)\right)=a\left(\ell_{r}\left(\bar{u}_{r}\right)\right)$ and $\bar{u}_{r}$ is a solution to $(\mathrm{P}-r)$ satisfying the desired estimates.

Proposition 4.7. If (4.1)-(4.5) hold and a is non decreasing on $\left[0, \ell_{L}\left(u_{0}\right)\right]$ then for any $r$ in $[0, L]$ and $u_{L}$ solution to $(P-L)$, there exists a solution $u_{r}$ to $(P-r)$ such that

$$
\frac{a\left(\ell_{L}\left(u_{L}\right)\right)}{a\left(\ell_{L}\left(u_{0}\right)\right)} u_{L} \leq u_{r} \leq u_{0} .
$$

Proof. If $v \in L_{\mathrm{r}}^{2}(\Omega)$ satisfies

$$
\frac{a\left(\ell_{L}\left(u_{L}\right)\right)}{a\left(\ell_{L}\left(u_{0}\right)\right)} u_{L} \leq v \leq u_{0}
$$

then, since $g, u_{0} \geq 0$, and $a$ is non decreasing on $\left[0, \ell_{L}\left(u_{0}\right)\right]$, we deduce

$$
a(0) \leq a\left(\ell_{r}(v)\right) \leq a\left(\ell_{r}\left(u_{0}\right)\right) \leq a\left(\ell_{L}\left(u_{0}\right)\right) .
$$

Proposition 4.2 yields

$$
u_{0} \geq T_{r}(v) \geq \frac{\varphi}{a\left(\ell_{L}\left(u_{0}\right)\right)} .
$$

Recall that $T_{r}$ is defined by $(2.3)$ and notice that $\varphi=a\left(\ell_{L}\left(u_{L}\right)\right) u_{L}$ for all solution $u_{L}$. We conclude with the Schauder fix point theorem.

In the sequel, we will assume that $a(0) \geq a\left(\mu_{L}\right)$ so that $u_{0} \leq u_{L}$. We obtain finer results because this assumption agree well with the monotonicity of $\ell_{r}$. For any $u, v$ in $L^{2}(\Omega)$, denote by $[u, v]$ the set

$$
\left\{w \in L^{2}(\Omega): u \leq w \leq v\right\} .
$$

Then we have the 
Proposition 4.8. Under the assumptions (4.1)-(4.5), assume in addition that

(i) there exits a solution $\mu_{L}$ to (3.5) with $a\left(\mu_{L}\right)=\min _{\left[0, \mu_{L}\right]}$ a;

(ii) $a(0)=\max _{\left[0, \mu_{L}\right]}$ a.

Then there exists a radial solution to $(P-r)$ in $\left[u_{0}, u_{L}\right]$ where $u_{L}:=\varphi / a\left(\mu_{L}\right)$.

Proof. We use a fix point argument in $\left[u_{0}, u_{L}\right] \cap L_{\mathrm{r}}^{2}(\Omega)$. If $v$ lies in this non empty closed and convex set then, by (4.5),

$$
0 \leq \ell_{r}(v) \leq \ell_{r}\left(u_{L}\right) \leq \ell_{L}\left(u_{L}\right)=\mu_{L} \quad \text { in } \Omega .
$$

Thus $(i)$, (ii) yield $a(0) \geq a\left(\ell_{r}(v)\right) \geq a\left(\mu_{L}\right)$. From Proposition 4.2, we infer

$$
u_{0} \leq T_{r}(v) \leq u_{L} .
$$

We conclude with the Schauder fix point theorem.

Definition 4.1. Being given $u:[0, L] \rightarrow H_{0}^{1}(\Omega)$, the graph of $u$ is called a (global) branch of solutions if

(i) $u \in C\left([0, L], H_{0}^{1}(\Omega)\right)$,

(ii) $u(r)$ is solution to $(P-r)$ for all $r$ in $[0, L]$.

If $u$ is defined only on a subinterval of $[0, L]$ with positive measure then we will speak about a local branch of solutions.

Definition 4.2 ([AP93]). Let $\{(r, u(r)): r \in[0, L]\}$ be a branch of solutions and $r_{0} \in[0, L]$. Then $\left(r_{0}, u\left(r_{0}\right)\right)$ is called a bifurcation point for this branch if there exists a sequence $\left(r_{n}, u_{n}\right)$ in $[0, L] \times H_{0}^{1}(\Omega)$ such that for all $n, u_{n}$ is a solution to $\left(P-r_{n}\right), u_{n} \neq u\left(r_{n}\right)$ and

$$
\left(r_{n}, u_{n}\right) \underset{n \rightarrow \infty}{\longrightarrow}\left(r_{0}, u\left(r_{0}\right)\right) \quad \text { in } \mathbb{R} \times H_{0}^{1}(\Omega) .
$$

Theorem 4.1. Under the assumptions and notation of Proposition 4.8, assume in addition that $a \in W^{1, \infty}(\mathbb{R})$ and, for some positive number $\delta \mu$, it holds that

$$
C_{1}\|g\|_{2}\|f\|_{*}\left\|a^{\prime}\right\|_{\infty,\left[-\delta \mu, \mu_{L}+\delta \mu\right]} \frac{1}{a\left(\mu_{L}\right)^{2}}<1,
$$

where $C_{1}$ is the constant of Lemma 2.1. Then

(i) for all $r$ in $[0, L],(P-r)$ possesses a unique radial solution $u_{r}$ in $\left[u_{0}, u_{L}\right]$;

(ii) $\left\{\left(r, u_{r}\right): r \in[0, L]\right\}$ is a branch of solutions without bifurcation point;

(iii) it is the only global branch of solutions;

(iv) if, in addition, $a$ is non increasing on $\left[0, \mu_{L}\right]$ then $r \mapsto u_{r}$ is non decreasing.

Proof. In view of Proposition 4.8, let $u_{r}$ be any radial solution to $(\mathrm{P}-r)$ lying in $\left[u_{0}, u_{L}\right]$ and $\bar{a}$ define by (4.18). Then $u_{r}$ is solution to

$$
-\operatorname{div}\left(\bar{a}\left(\ell_{r}(u)\right) \nabla u\right)=f \quad \text { in } H^{-1}(\Omega), \quad u \in H_{0}^{1}(\Omega) .
$$

According to (4.19) and $\bar{a} \geq a\left(\mu_{L}\right), \bar{a}$ satisfies (2.13) so that, by Corollary 2.2, the above problem is uniquely solvable. Thus we have proved the first assertion (i) of the theorem. 
The solution to (4.20) satisfies (2.4) with $a=\bar{a}$. Moreover, the positivity of $g$ and $u_{0}$ yields

$$
0 \leq \ell_{r}\left(u_{r}\right) \leq \ell_{r}\left(u_{L}\right) \leq \ell_{L}\left(u_{L}\right)=\mu_{L}
$$

Thus, by Corollary 2.1, $\left\{\left(r, u_{r}\right): r \in[0, L]\right\}$ is a branch of solutions for the original problems. Besides, Theorem 2.3 prevents the existence of bifurcation point. In order to prove (iii), let $\left\{\left(r, v_{r}\right): r \in[0, L]\right\}$ be a branch of solutions. The set

$$
\left\{r \in[0, L]: u_{r}=v_{r}\right\}
$$

is closed since $u_{r}-v_{r}$ is continuous. Moreover, $u_{r}$ satisfies $(2.14)$ for $\mu_{1}=\mu_{L}$ hence this set is also open according to Theorem 2.3. By connexity, it is empty or equal to $[0, L]$. Since $u_{0}=v_{0}$, it must be equal to $[0, L]$. This proves the uniqueness of global branch.

There remains to prove that $\left(u_{r}\right)$ is non decreasing. For this let $r, s \in[0, L]$, $r<s$ and $v$ in $\left[u_{r}, u_{L}\right] \cap L_{\mathrm{r}}^{2}(\Omega)$. Then

$$
\ell_{r}\left(u_{r}\right) \leq \ell_{s}(v) \leq \ell_{L}\left(u_{L}\right)
$$

and since $a$ is non increasing, we deduce $a\left(\ell_{r}\left(u_{r}\right)\right) \geq a\left(\ell_{s}(v)\right) \geq a\left(\ell_{L}\left(u_{L}\right)\right)$. By Proposition 4.2, it follows that $u_{r} \leq T_{r}(v) \leq u_{L}$. Thus the Schauder fix point theorem provides a solution to $(\mathrm{P}-s)$ belonging to $\left[u_{r}, u_{L}\right] \cap L_{\mathrm{r}}^{2}(\Omega)$. By the uniqueness result in $\left[u_{0}, u_{L}\right]$, this solution is $u_{s}$. Thus $u_{r} \leq u_{s}$.

Corollary 4.2. Suppose that $f, g$ satisfy (4.1), (4.2), (4.4), (4.5) and $g$ can be extended outside of $\Omega$ by a function belonging to $H^{1}\left(\mathbb{R}^{n}\right)$. Then there exist functions $a(\cdot)$ satisfying $(3.7)$ and $u_{L}^{2}$ solution to $(P-L)$ with the following property. There exist at least a local branch of solutions starting from $u_{L}^{2}$ but there is no global branch starting from $u_{L}^{2}$.

Proof. It is clear that we can construct functions $a$ satisfying (3.7) and the following conditions (see Fig. 3).

(i) There exits a solution $\mu_{1}$ to $(3.5)$ with $a\left(\mu_{1}\right)=\min _{\left[0, \mu_{1}\right]} a$.

(ii) $a(0)=\max _{\left[0, \mu_{1}\right]} a$.

(iii) $C_{1}\|g\|_{2}\|f\|_{*}\left\|a^{\prime}\right\|_{\infty,\left[0, \mu_{1}\right]} \frac{1}{a\left(\mu_{1}\right)^{2}}<1$.

(iv) There exists $\mu_{2}>\mu_{1}$ solution to (3.5) with $\mu_{2} a^{\prime}\left(\mu_{2}\right) \neq a\left(\mu_{2}\right)$.

By Theorem 3.1, for $i=1,2, u_{i}:=\varphi / a\left(\mu_{i}\right)$ is solution to $(\mathrm{P}-L)$ and $\mu_{i}=\ell_{L}\left(u_{i}\right)$. By (iv), Theorem 3.2 and the implicit function theorem, there is a local branch of solutions starting from $u_{2}$.

Theorem 4.1 yields the existence of a unique global branch of solutions and this branch passes through $u_{1}$. Since $\mu_{2}>\mu_{1}$, we deduce that $u_{1} \neq u_{2}$. Hence there is no global branch starting from $u_{2}$.

\section{REFERENCES}

[AP93] Antonio Ambrosetti and Giovanni Prodi. A primer of nonlinear analysis, volume 34 of Cambridge Studies in Advanced Mathematics. Cambridge University Press, Cambridge, 1993.

[CCR06] Emmanuel Chasseigne, Manuela Chaves, and Julio D. Rossi. Asymptotic behavior for nonlocal diffusion equations. J. Math. Pures Appl. (9), 86(3):271-291, 2006. 


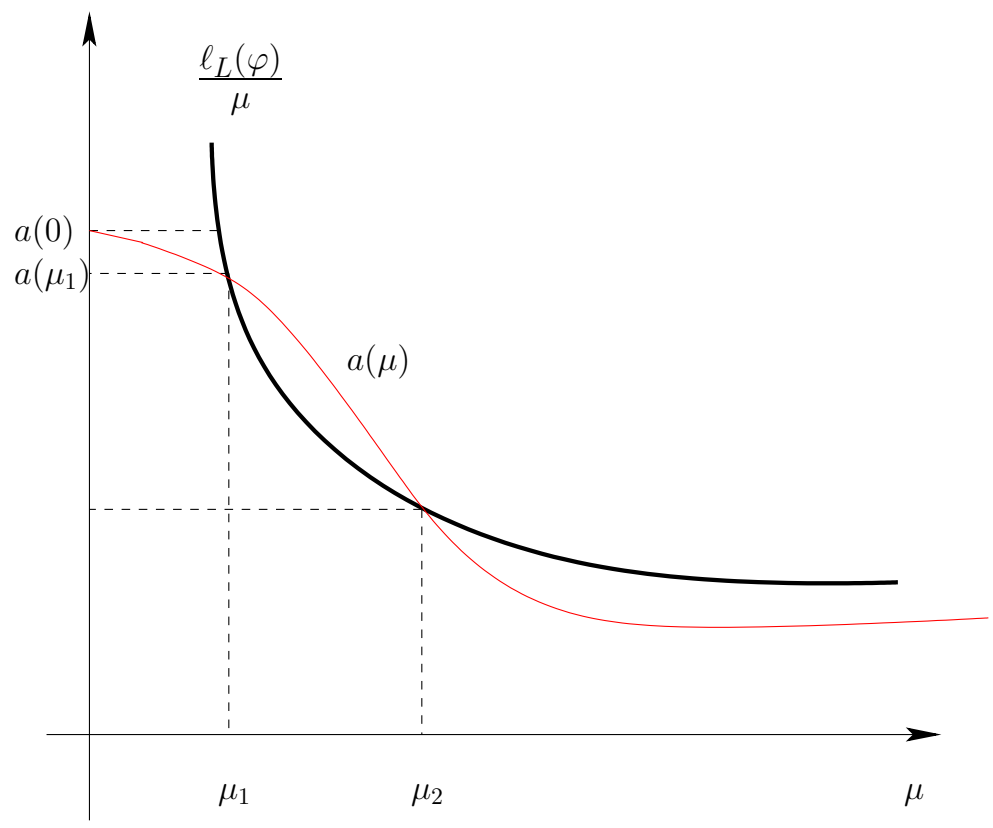

Figure 3

[CL99] M. Chipot and B. Lovat. On the asymptotic behaviour of some nonlocal problems. Positivity, 3(1):65-81, 1999.

[CM01] Michel Chipot and Luc Molinet. Asymptotic behaviour of some nonlocal diffusion problems. Appl. Anal., 80(3-4):279-315, 2001.

[ZP06] Zhitao Zhang and Kanishka Perera. Sign changing solutions of Kirchhoff type problems via invariant sets of descent flow. J. Math. Anal. Appl., 317(2):456-463, 2006.

\section{Laboratoire de Mathématiques et Applications}

\section{UMR 6086}

Université DE Poitiers \& CNRS

SP2MI - BP 30179

86962 Futuroscope Chasseneuil Cedex - France

E-mail address: Armel.Andami-Ovono@math.univ-poitiers.fr

E-mail address: Arnaud.Rougirel@math.univ-poitiers.fr 